\title{
$\dot{\nabla}$ Global Clinical Fesearch Journal
}

\section{Os benefícios do aleitamento materno na primeira hora de vida}

The benefits of breastfeeding in the first hour of life

Los beneficios de la lactancia materna en la primera hora de vida

\author{
Nathalia Cristina Machado Prado Ramiro ${ }^{1^{*}}$ \\ ORCID: 0000-0002-6949-6134 \\ Mariana de Souza Pereira ${ }^{1}$ \\ ORCID: 0000-0002-8384-8538 \\ Rafaela Silva de Souza ${ }^{1}$ \\ ORCID: 0000-0001-8776-9506
}

\author{
Bianca Regina de Michelli Chaparin ${ }^{1}$ \\ ORCID: 0000-0002-8942-7259 \\ Bruna Vitória Aguiar Navarro ${ }^{1}$ \\ ORCID: 0000-0002-2030-0823 \\ Luciane Andrea Aver ${ }^{2}$ \\ ORCID: 0000-0002-2432-7628
}

\footnotetext{
${ }^{1}$ Centro Universitário São Camilo. São Paulo, Brasil.

${ }^{2}$ Universidade Federal de São Paulo. São Paulo, Brasil.

*Autor correspondente: E-mail: ramironathalia425@gmail.com
}

\section{Resumo}

Objetivou-se identificar na literatura científica a importância do aleitamento materno na primeira hora de vida do recém-nascido. Trata-se de uma pesquisa de revisão integrativa da literatura, elaborada a partir da questão norteadora "Qual a importância do aleitamento materno na primeira hora de vida?". Foram realizadas pesquisas nas bases de dados LILACS, SciELO, Medline e BDENF, utilizando os descritores "Aleitamento materno", "Período pós-parto", "Maternidades", "Serviços de saúde materno-infantil" e "Recém-nascido". Utilizando os critérios de inclusão: artigos no idioma português, publicados com intervalo dos últimos cinco anos, disponíveis totalmente na integra através do acesso eletrônico. E os critérios de exclusão: dissertações, duplicações, que não respondiam à questão norteadora e artigos de revisão. O aleitamento materno na primeira hora de vida gera a redução do índice de mortalidade neonatal, proteção imunológica e promove condições para o desenvolvimento de sistemas do corpo do recém-nascido, além de produzir a ocitocina, reduzindo o risco de se desenvolver hemorragia e outras complicações maternas. Nota-se que o aleitamento materno na primeira hora de vida do recém-nascido oferece inúmeros benefícios maternos e principalmente ao neonato, promovendo melhoria na saúde global da criança.

Descritores: Aleitamento Materno; Período Pós-Parto; Maternidades; Serviços de Saúde Materno-Infantil; Recém-Nascido.

\section{Como citar este artigo:}

Ramiro NCMP, Pereira MS, Souza RS, Chaparin BRM, Navarro BVA, Aver LA. Os benefícios do aleitamento materno na primeira hora de vida. Glob Clin Res. 2021;1(1):e7.

Editor Chefe: Caroliny dos Santos Guimarães da Fonseca

Editor Executivo: Kátia dos Santos Armada de Oliveira 
The aim was to identify in the scientific literature the importance of breastfeeding in the newborn's first hour of life. This is an integrative literature review, based on the guiding question "What is the importance of breastfeeding in the first hour of life?". Searches were carried out in LILACS, SciELO, Medline and BDENF databases, using the descriptors "Breastfeeding", "Postpartum period", "Maternity Hospitals", "Maternal and Child Health Services" and "Newborn". Using the inclusion criteria: articles in Portuguese, published at interval of the last five years, fully available through electronic access. And the exclusion criteria: dissertations, duplications, which did not answer the guiding question and review articles. Breastfeeding in the first hour of life reduces the neonatal mortality rate, immunological protection and promotes conditions for the development of the newborn's body systems, in addition to producing oxytocin, reducing the risk of developing hemorrhage and other maternal complications. It is noted that breastfeeding in the first hour of the newborn's life offers numerous maternal benefits and especially for the newborn, promoting an improvement in the child's overall health.

Descriptors: Breastfeeding; Postpartum Period; Maternity Wards; Maternal and Child Health Services; Newborn.

\section{Resumén}

El objetivo fue identificar en la literatura científica la importancia de la lactancia materna en la primera hora de vida del recién nacido. Se trata de una revisión integradora de la literatura, basada en la pregunta orientadora "¿Cuál es la importancia de la lactancia materna en la primera hora de la vida?". Las búsquedas se realizaron en las bases de datos LILACS, SciELO, Medline y BDENF, utilizando los descriptores "Lactancia materna", "Posparto", "Hospitales de maternidad", "Servicios de salud maternoinfantil" y "Recién nacido". Utilizando los criterios de inclusión: artículos en portugués, publicados a intervalo de los últimos cinco años, totalmente disponibles a través de acceso electrónico. Y los criterios de exclusión: disertaciones, duplicaciones, que no respondieron a la pregunta orientadora y artículos de revisión. La lactancia materna en la primera hora de vida reduce la tasa de mortalidad neonatal, la protección inmunológica y promueve las condiciones para el desarrollo de los sistemas corporales del recién nacido, además de producir oxitocina, reduciendo el riesgo de desarrollar hemorragias y otras complicaciones maternas. Se observa que la lactancia materna en la primera hora de vida del recién nacido ofrece numerosos beneficios para la madre y especialmente para el recién nacido, promoviendo una mejora en la salud general del niño.

Descriptores: Amamantamiento; Período Posparto; Hospitales de Maternidad; Servicios de Salud Maternoinfantil; Recién Nacido.

\section{Introdução}

A amamentação é considerada uma estratégia importante de sobrevivência infantil pelo Fundo das Nações Unidas para a Infância (Unicef), pela Organização Mundial da Saúde (OMS) e por órgãos de proteção à criança. Na primeira hora pós-parto, a amamentação se constitui na primeira prática alimentar do ser humano, sendo considerada um dos fatores que garantirá a sua saúde, o seu crescimento e desenvolvimento adequados, em todas as fases do ciclo da vida. Além de conferir vantagens para as crianças, mulheres e para a sociedade, representa a mais sensível, econômica e eficaz intervenção para a promoção da saúde maternoinfantil ${ }^{1-3}$.

No primeiro dia de vida, a amamentação reduz em $16,0 \%$ as chances de mortes neonatais. $E$, quando iniciada até a primeira hora de vida, a porcentagem dessa redução dessa mortalidade passa para $22,0 \%$, sendo que quanto mais se prorroga o início do aleitamento materno, maiores as chances de mortalidade neonatal causadas por infecções ${ }^{2}$.

O leite materno proporciona uma nutrição de alta qualidade concedendo todos os nutrientes necessários para a criança devido as suas propriedades nutricionais. Contém em sua composição: colostro, proteínas, gorduras, carboidratos, vitaminas, minerais, oligoelementos e componentes imunológicos que proporcionam efeito protetor levando a um aumento da imunidade do lactente e benefícios que se estenderão ao longo da vida ${ }^{4}$.

São inúmeros os benefícios imediatos do aleitamento materno exclusivo logo após o parto. Para o recém-nascido, o colostro conhecido como a "primeira vacina" garante a capacidade contra infecções, como por exemplo, para enterocolite necrosante, infecções do trato gastrointestinal e respiratório, alergias, septicemia e meningites, além de estar sempre pronto na temperatura ideal para a criança. O aleitamento após o parto também é benéfico para a mãe, já que a sucção estimula a liberação de ocitocina endógena, que induz a contração uterina, prevenindo hemorragias puerperais, principal causa de mortalidade materna no mundo ${ }^{1}$.

O contato pele a pele, logo após o nascimento, favorece o estabelecimento do vínculo mãe-filho, acarretando benefícios físicos e psíquicos para ambos. Com este contato, o bebê se mantém aquecido por meio do calor do corpo da mãe, o que evita a hipotermia, auxilia na adaptação da transição fetal-neonatal e favorece a colonização do intestino do recém-nascido por microrganismos da flora cutânea materna, conferindo ao neonato maior imunidade ${ }^{1}$. 
Os benefícios do aleitamento materno na primeira hora de vida

Nesse contexto, a forma mais segura, eficaz e completa de alcançar o crescimento e desenvolvimento adequados de uma criança é garantindo o aleitamento materno desde a primeira hora de vida extrauterina ${ }^{1}$.

Objetivou-se identificar na literatura científica a importância do aleitamento materno na primeira hora de vida do recém-nascido.

\section{Metodologia}

Trata-se de uma pesquisa de revisão integrativa da literatura, sendo realizada cumprindo as seis etapas de elaboração, são elas: 10 Identificação do tema e seleção de hipóteses ou questão de pesquisa para a elaboração da revisão integrativa; 20 estabelecimento de critérios para a inclusão e exclusão de estudos/amostragem ou pesquisa de literatura; 3o definição das informações a serem extraídas dos estudos; 4 ㅇ avaliação dos estudos incluídos na revisão integrativa; 50 interpretação dos resultados; 6ㅇ apresentação da revisão/síntese do conhecimento ${ }^{5}$.

Etapa 1: Identificação do tema e seleção de hipóteses ou questão de pesquisa para a elaboração da revisão integrativa

A revisão integrativa começa a partir da definição de um problema e a formulação de uma hipótese ou questão de pesquisa que apresente relevância para a área que está sendo estudada. Essa etapa é considerada norteadora para uma revisão integrativa bem elaborada, pelo fato dela estar associada a um raciocínio logico, e o assunto deve ser definido de forma clara e objetiva, fazendo com que a análise seja realizada por completo. A questão bem definida, facilitara a definição dos descritores e a procura dos estudos relacionados ${ }^{5}$

Etapa 2: Estabelecimento de critérios para inclusão e exclusão de estudos/amostragem ou pesquisa da literatura Está etapa está diretamente relacionada com a etapa anterior, em que é realizado uma pesquisa em bases de dados de forma ampla e diversificada, pesquisando em bases eletrônicas, busca manual em periódicos, referências descritas nos estudos selecionados, contato com pesquisadores e a utilização de material não publicado. Fazendo com que tenha uma validade na pesquisa, permitindo que as conclusões sejam confiáveis e amplas. Dessa forma, todas as decisões tomadas têm as considerações dos critérios de inclusão e exclusão dos estudos, sendo justificadas na metodologia da revisão. Nesta fase da revisão integrativa da literatura deve ser totalmente documentada, incluindo os critérios de inclusão e exclusão, descritores utilizados, bases de dados das consultas, estratégias de pesquisa, para que seja possível determinar as pesquisas primárias relevantes ${ }^{5}$.

Etapa 3: Definição das informações a serem extraídas dos estudos selecionados/ categorização dos estudos

Esta etapa consiste na definição das informações a serem extraídas dos estudos que foram selecionados, utilizando-se um instrumento para reunir e sintetizar os instrumentos chave. As evidências dos estudos selecionados
Ramiro NCMP, Pereira MS, Souza RS, Chaparin BRM, Navarro BVA, Aver LA devem ser avaliadas a fim de determinar a confiança do uso dos resultados e fortalecer as conclusões que chegaram no conhecimento do tema que está sendo estudado. O pesquisador deve organizar e sumarizar as informações, gerando um banco de dados de fácil acesso e manejo ${ }^{5}$.

\section{Etapa 4: Avaliação dos estudos incluídos na revisão} integrativa da literatura

Nesta etapa é realizado a análise dos dados, semelhante a análise dos dados de uma investigação convencional. No sentido de garantir a validação da revisão, os estudos selecionados devem ser analisados rigorosamente e detalhadamente, procurando as explicações para os resultados contraditórios ou diferentes ${ }^{5}$.

\section{Etapa 5: Interpretação dos resultados}

Esta etapa consiste a discussão dos principais resultados da pesquisa convencional, em que o pesquisador realiza uma comparação entre os resultados da avaliação crítica dos estudos, incluindo o conhecimento teórico e destacando as conclusões e implicações resultantes da revisão integrativa. Com isso, é possível identificar as lacunas existentes, permitindo com que sejam levantadas sugestões para pesquisas futuras ${ }^{5}$.

Etapa 6: Apresentação da revisão/síntese do conhecimento Nesta etapa é elaborado um documento que deve conter a descrição das etapas percorridas pelo pesquisador e as principais evidencias que foram encontradas durante a análise dos estudos ${ }^{5}$.

A revisão integrativa tem o objetivo de apresentar informações suficientes, de forma com que o leitor possa avaliar a pertinência dos procedimentos utilizados na realização da revisão, dos tópicos abordados e da descrição dos estudos incluídos ${ }^{5}$.

Dessa maneira, a pesquisa de revisão integrativa foi desenvolvida a partir da pergunta norteadora: "Qual a importância do aleitamento materno na primeira hora de vida do recém-nascido?".

Realizada através de pesquisas online nas bases de dados LILACS, SciELO, Medline e BDENF, utilizando os descritores "Aleitamento materno", "Período pós-parto", "Maternidades", "Serviços de saúde materno-infantil" e "Recém-nascido".

Após realizar o levantamento de dados, foram utilizados os critérios de inclusão para a realização este estudo: artigos no idioma português, publicados com intervalo dos últimos cinco anos, disponíveis totalmente na integra através do acesso eletrônico. E os critérios de exclusão: dissertações, duplicações, que não respondiam à questão norteadora e artigos de revisão.

Com isso, os artigos foram pré-selecionados durante as buscas nas bases de dados, através de uma leitura objetiva e superficial, buscando somente os estudos que estavam dentro da temática e que atendessem aos critérios de inclusão e exclusão que já estavam estabelecidos. Após foi realizado uma leitura mais detalhada e rigorosa dos estudos, com o objetivo de selecionar os que respondiam à questão norteadora e atendessem aos critérios de inclusão. 
Os benefícios do aleitamento materno na primeira hora de vida

Dessa maneira, foram selecionados 12 artigos que Ramiro NCMP, Pereira MS, Souza RS, Chaparin BRM, Navarro BVA, Aver LA estavam correspondentes com a temática abordada.

Figura 1. Fluxograma de identificação, triagem, elegibilidade e inclusão de referências. São Paulo, SP, Brasil, 2020
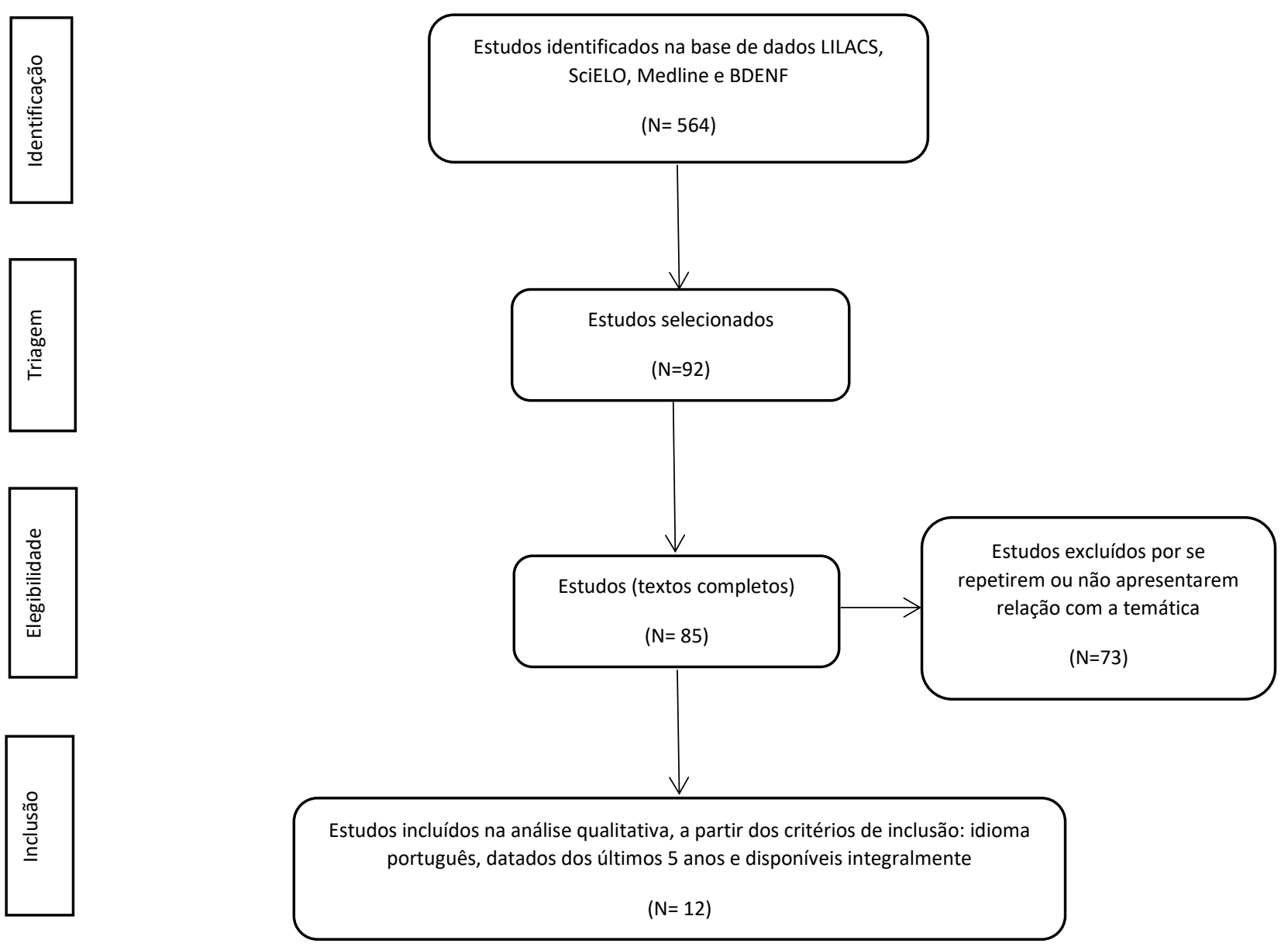

\section{Resultados}

Pode-se observar na Figura 2, o quadro sinóptico dos artigos selecionados para a elaboração da presente revisão integrativa, contemplando as seguintes variáveis: ano de publicação, autores, título e objetivo(s). E na Figura 3 , observa-se a presença de quadro sinóptico dos artigos selecionados para a composição do estudo contendo o título e a síntese dos resultados.

Figura 2. Quadro sinóptico dos artigos selecionados contendo ano de publicação, autor e objetivos. São Paulo, SP, Brasil, 2020

\begin{tabular}{|c|c|c|c|}
\hline $\begin{array}{c}\text { ANO DE } \\
\text { PUBLICAÇÃO }\end{array}$ & AUTORES & TÍTULO & OBJETIVO (S) \\
\hline Agosto/2016 & $\begin{array}{l}\text { LEITE, Maura Fernanda } \\
\text { Ferreira da Silva; BARBOSA, } \\
\text { Priscila Araújo; OLIVINDO, } \\
\text { Dean Douglas Ferreira de; } \\
\text { XIMENEZ, Valessa de } \text { Lima. }^{9}\end{array}$ & $\begin{array}{l}\text { Promoção do aleitamento } \\
\text { materno na primeira hora de } \\
\text { vida do recém-nascido por } \\
\text { profissionais da enfermagem. }\end{array}$ & $\begin{array}{l}\text { Descrever e analisar a percepção das puérperas } \\
\text { acerca do incentivo ao aleitamento materno na } \\
\text { primeira hora de vida pelos profissionais de } \\
\text { enfermagem em uma maternidade pública. }\end{array}$ \\
\hline Junho/2016 & $\begin{array}{l}\text { SAMPAIO, Ádila Roberta } \\
\text { Rocha; BOUSQUAT, Aylene; } \\
\text { BARROS, Claudia. }{ }^{13}\end{array}$ & $\begin{array}{l}\text { Contato pele a pele ao nascer: } \\
\text { um desafio para a promoção do } \\
\text { aleitamento materno em } \\
\text { maternidade pública no } \\
\text { Nordeste brasileiro com o título } \\
\text { de Hospital Amigo da Criança. }\end{array}$ & $\begin{array}{l}\text { Identificar a prevalência do cumprimento do quarto } \\
\text { passo da Iniciativa Hospital Amigo da Criança (IHAC) } \\
\text { - colocar os bebês em contato pele a pele com suas } \\
\text { mães imediatamente após o parto, por no mínimo } \\
\text { meia hora - em uma maternidade pública do } \\
\text { Nordeste brasileiro. }\end{array}$ \\
\hline $\begin{array}{l}\text { Novembro/ } \\
2017\end{array}$ & $\begin{array}{l}\text { ROCHA, Letícia Braga; } \\
\text { ARAÚJO, Fellipe Marques da } \\
\text { Silva; ROCHA, Naira Coutinho } \\
\text { Oliveira; ALMEIDA, } \\
\text { Cristiano Dantas de; SANTOS, } \\
\text { Miriam Oliveira dos; ROCHA, } \\
\text { Carlos Henrique Roriz da. }{ }^{6}\end{array}$ & $\begin{array}{l}\text { Aleitamento materno na } \\
\text { primeira hora de vida: uma } \\
\text { revisão da literatura. }\end{array}$ & $\begin{array}{l}\text { Destacar os benefícios do aleitamento materno na } \\
\text { primeira hora de vida, em relação à taxa de } \\
\text { morbimortalidade infantil. }\end{array}$ \\
\hline
\end{tabular}


Os benefícios do aleitamento materno na primeira hora de vida

Fevereiro/ 2017 ANTUNES, Marcos Benatti; DEMITTO, Marcela de Oliveira; SOARES, Larissa Gramazio; RADOVANOVIC Cremilde Aparecida Trindade; HIRAGASHI, leda Harumi; ICHISATO, Sueli Mutsumi Tsukuda; PELLOSO Sandra Marisa. ${ }^{14}$

\begin{tabular}{|c|c|}
\hline $\begin{array}{l}\text { Setembro/ } \\
2017\end{array}$ & $\begin{array}{l}\text { SANTOS, Rayra Pereira Buriti; } \\
\text { ARAÚJO, Rosália Teixeira; } \\
\text { TEIXEIRA, Marizete Argolo; } \\
\text { RIBEIRO, Vívian Mara; LOPES, } \\
\text { Alana } \\
\text { Silva; ARAÚJO, Vanessa } \\
\text { Matos. }\end{array}$ \\
\hline Janeiro/2019 & $\begin{array}{l}\text { SILVA, Juliane Lima Pereira } \\
\text { da; LINHARES, Francisca } \\
\text { Márcia Pereira; BARROS, } \\
\text { Amanda de Almeida; SOUZA, } \\
\text { Auricarla Gonçalves de; } \\
\text { ALVES, Danielle Santos; } \\
\text { ANDRADE, Pryscila de }\end{array}$ \\
\hline
\end{tabular}

\begin{tabular}{l} 
Novembro/ \\
2019 \\
\\
\hline Junho/2020
\end{tabular}

Oliveira Nascimento. ${ }^{10}$

\begin{tabular}{lll}
\hline Outubro/2020 & $\begin{array}{l}\text { JUNG, Silvana } \\
\text { RODRIGUEZ, } \\
\text { Araujo; }\end{array}$ & Mendes; \\
& Fernand
\end{tabular}

Novembro/ $\quad$ JESUS, Alyne Santana de; 2020

\begin{tabular}{|c|c|}
\hline & \\
\hline Fevereiro/2020 & $\begin{array}{l}\text { SENA, Rafaela Pereira de; } \\
\text { SOUZA, Gabriela Nascimento } \\
\text { de; MONTEIRO, Luiza } \\
\text { Alessandra Oliveira; SOUZA, } \\
\text { Yenne Patrícia Rodrigues de; } \\
\text { SANTOS, Yves de Luka } \\
\text { Miranda dos; ARAÚJO, Maria } \\
\text { Rute de Souza; SILVA, Silvia } \\
\text { Cristina Santos da; SILVA, } \\
\text { Rodrigo Maia da; PARENTE, } \\
\text { Jorgeany Soares. }{ }^{11}\end{array}$ \\
\hline Novembro/2020 & $\begin{array}{l}\text { TERRA, Nathália Oliveira; } \\
\text { GÓES, Fernanda Garcia } \\
\text { Bezerra; SOUZA, Andressa } \\
\text { Neto; LEDO, Beatriz Cabral; } \\
\text { CAMPOS, Brenda Lucas; } \\
\text { BARCELLOS, Thamires } \\
\text { Myrena Torres. }{ }^{17}\end{array}$ \\
\hline
\end{tabular}

Ramiro NCMP, Pereira MS, Souza RS, Chaparin BRM, Navarro BVA, Aver LA Amamentação na primeira hora $\quad$ Verificar o conhecimento e prática sobre a de vida: conhecimento e prática da equipe multiprofissional. amamentação na primeira hora de vida entre membros da equipe multiprofissional de um hospital do município de Maringá, Paraná.

\section{Importância do colostro para a saúde do recém-nascido: percepção das puérperas. \\ Fatores associados ao aleitamento materno na \\ Compreender a percepção das puérperas quanto à importância do colostro para a saúde do recém- nascido e realizar cuidado educativo às puérperas e seus familiares quanto à importância do colostro. \\ Avaliar os fatores associados à prática do aleitamento materno na primeira hora pós-parto.} primeira hora de vida em um Hospital Amigo da Criança.
RAMALHO, Alanderson Alves; MARTINS, Fernanda Andrade; LIMA, Thaíla Alves dos Santos; ANDRADE, Andréia Moreira, KOIFMAN, Rosalina Jorge. ${ }^{12}$ RODRIGUES, Cristina dos Santos de Freitas; SANTOS, Bianca Zimmerman dos; LIPINSKI, Jussara; COSTENARO, Regina Gema Santini; ZAMBERLAN, Cláudia. ${ }^{15}$ HERBER, Silvani. ${ }^{16}$

Fatores associados à amamentação na primeira hora de vida em Rio Branco, Acre.

Aleitamento materno exclusivo na primeira hora de vida: uma revisão integrativa.

Contato pele a pele e aleitamento materno: experiências de puérperas

Amamentação na primeira hora de vida entre mulheres do Nordeste brasileiro: Ferreira; SANTOS, José Marcos de Jesus; FREITAS, Carla Kalline Alves Cartaxo; MENDES, Rosemar Barbosa; LEITE, Adriana Moraes; REITE, Adriana Moraes; Campos Verdes. Ação educativa para as gestantes na promoção da "Golden Hour": relato de experiência.
Determinar a prevalência e analisar os fatores associados à amamentação na primeira hora de vida em Rio Branco, Acre.

Investigar as evidências científicas sobre aleitamento materno exclusivo na primeira hora de vida.

Descrever as experiências de puérperas quanto ao contato pele a pele com o recém-nascido, realizado na primeira hora de vida e o início do aleitamento materno.

Identificar a prevalência e os fatores associados à amamentação na primeira hora de vida.

Relatar a experiência vivenciada pela atividade educativa para gestantes na promoção da Golden hour, realizada em uma Estratégia Saúde da Família (ESF), no município de Belém-PA.
Fatores intervenientes na adesão à amamentação na primeira hora de vida: revisão integrativa.
Analisar evidências científicas na área da saúde sobre os fatores intervenientes na amamentação do recém-nascido na primeira hora de vida no ambiente hospitalar. 


\begin{abstract}
Promoção do aleitamento materno na primeira hora de Vida do recém-nascido por profissionais da enfermagem.

Contato pele a pele ao nascer: um desafio para a promoção do aleitamento materno em maternidade pública no Nordeste brasileiro com o título de Hospital Amigo da Criança.
\end{abstract}

Aleitamento materno na primeira hora de vida: uma revisão da literatura.

Amamentação na primeira hora de vida: conhecimento e prática da equipe multiprofissional.

Importância do colostro para a saúde do recémnascido: percepção das puérperas.

Fatores associados ao aleitamento materno na primeira hora de vida em um Hospital Amigo da Criança.

Fatores associados à amamentação na primeira hora de vida em Rio Branco, Acre.

Aleitamento materno exclusivo na primeira hora de vida: uma revisão integrativa.

Contato pele a pele e aleitamento materno: experiências de puérperas.

Amamentação na primeira hora de vida entre mulheres do Nordeste brasileiro: prevalência e fatores associados.

\section{Ação educativa para as gestantes na promoção} da "Golden Hour": relato de experiência.

\section{Fatores intervenientes na adesão à} amamentação na primeira hora de vida: revisão integrativa.
Evidenciou-se a percepção das gestantes em relação aos benefícios do aleitamento maternos, tais como: sentimentos como afeto e amor, fortalecimento de vínculo entre o binômio e a importância do colostro como protetor imunológico.

Observou-se baixa adesão ao quarto passo da IHAC em todo o território nacional, principalmente na região Nordeste, na qual os recém-nascidos apresentam poucas chances de serem amamentados na primeira hora de vida, tal fato está associado ao índice exorbitante do número de cesáreas e a ausência de informações durante o prénatal à gestante sobre amamentação.

A amamentação na primeira hora de vida auxilia no vínculo mãe-bebê e na prolongação da amamentação, bem como atua promovendo fator protetor advindo do colostro com potencial para reduzir cerca de $22 \%$ da mortalidade neonatal, garantindo melhoria na condição da saúde global da criança.

Os profissionais de saúde entrevistados demonstraram reconhecer os benefícios do aleitamento materno precoce, porém apresentam dificuldades em aderir tal ato por motivos de: prevalência de parto cesariano, redução de profissionais, ausência de alojamento conjunto, cuidados de enfermagem fragmentados o que distancia a mãe do recém-nascido.

O colostro exerce papel de suprir as carências do neonato, pois ele é composto por vitaminas lipossolúveis como $\mathrm{E}, \mathrm{A}$ e $\mathrm{K}$, proteínas, cálcio, potássio, além de promover a produção de anticorpos sendo descrito e reconhecido pelas puérperas como vacina natural.

O aleitamento materno ainda na sala de parto auxilia na regulação cardiorrespiratória, térmica, dos níveis glicêmicos e na adaptação extrauterina do recém-nascido. Além da sucção precoce estimular na liberação de ocitocina e prolactina, aumentando a produção de leite. Identificou-se a presença de enfermeiro em sala de parto, peso igual ou superior a 3000 gramas e contato pele a pele como fatores auxiliadores da amamentação na primeira hora de vida.

A prática do aleitamento materno na primeira hora de vida auxilia na promoção, proteção e apoio ao aleitamento materno. Evidenciou-se que os principais fatores de risco associados a não amamentação imediata após o parto são a cesárea e o baixo peso ao nascer.

Identificou-se que o aleitamento materno na primeira hora de vida favorece o estreitamento de laços afetivos entre a puérpera e seu recém-nascido. Além disso, a amamentação auxilia na adaptação extrauterina do bebê.

Abordou-se a importância do aleitamento materno imediato como forma de estabelecer vínculo entre o binômio, imunidade e melhoria no estado nutricional do bebê, bem como auxiliar nas condições físicas e psíquicas materna. Entretanto, estudos apontam que tal ato não é incentivado e tão pouco realizado.

Fatores como o desejo de ter um filho, realização de pré-natal adequado e orientação prévia acerca dos benefícios da amamentação favorecem o início do aleitamento materno na primeira hora de vida. Enquanto, pré-natal inadequado, cesariana e ausência de alojamento conjunto favorecem a amamentação tardia.

Em metodologia ativa abordou-se os princípios da "Golden Hour", incluindo o clampeamento oportuno e contato pele a pele, enfatizando a importância do aleitamento materno na primeira hora de vida, pois oferece nutrientes e previne o recém-nascido contra doenças infecciosas.

A amamentação precoce aumenta a frequência e a duração do aleitamento materno exclusivo, reduz a mortalidade infantil e caracteriza-se como o quarto passo da Iniciativa do Hospital Amigo da Criança. Porém, fatores maternos e neonatais, práticas institucionais e profissionais influenciam diretamente a amamentação na primeira hora de vida.
Com a análise e leitura minuciosa dos artigos selecionados para a composição e execução da revisão integrativa, pode-se construir quadro sinóptico contendo as variáveis de maior importância (Figura 2 e 3). Na qual, a partir da síntese dos resultados apresentados nos estudos analisados foi possível identificar quais são os benefícios do aleitamento materno na primeira hora de vida do recémnascido.

\section{Discussão}

A partir da análise dos estudos selecionados podese observar que os benefícios do aleitamento materno na primeira hora de vida são inúmeros e comprovados cientificamente. De acordo com estudo ${ }^{6}$, a amamentação precoce oferece ao binômio mãe-bebê diversas vantagens como, por exemplo a redução considerável do índice de mortalidade neonatal, desta forma garantindo a melhoria na saúde global da criança. Segundo autores ${ }^{7}$, o Aleitamento Materno na Primeira Hora de Vida é destacado e recomendado pela OMS, pois se trata da capacidade da interação entre os recém-nascidos (RN) com suas mães nos primeiros minutos de vida e faz parte das estratégias prioritárias para a promoção, proteção e apoio ao aleitamento materno. 
Os benefícios do aleitamento materno na primeira hora de vida

O colostro também conhecido como o primeiro leite que o recém-nascido tem contato ao realizar a sucção da mama de sua nutriz, caracteriza-se por ser um leite viscoso e amarelado, proveniente dos alvéolos. Tal qual possui diversas propriedades necessárias e que suprem as necessidades do bebê. Em sua composição encontra-se vitaminas lipossolúveis como $\mathrm{E}, \mathrm{A}$ e $\mathrm{K}$, riqueza de proteínas, além de conter sódio, cálcio e potássio. Ressalta-se a proteção imunológica advinda do colostro que confere a produção de anticorpos, sendo reconhecido por muitas gestantes e profissionais como "vacina natural"6,8.

Entre os benefícios psíquicos pode-se pontuar o estabelecimento do vínculo materno-infantil e a troca de sentimentos afetuosos como amor, carinho e confiança entre ambos, o que vem a auxiliar na prolongação do aleitamento materno de forma exclusiva posteriormente. Já entre os benefícios fisiológicos, observa-se que a amamentação ainda na primeira hora de vida, valorizando a "Golden Hour". Além de auxiliar na adaptação extrauterina do recém-nascido por promover a regulação glicêmica e auxiliar no sistema cardiorrespiratório ${ }^{9-11}$.

Ressalta-se que, quando o RN realiza a sucção da mama, ocorre o estímulo para a produção de ocitocina pelo hipotálamo, a ocitocina consiste em um hormônio que ajuda na contração uterina, reduzindo assim a perda sanguínea materna após o parto e, consequentemente, tenha menor risco de desenvolver hemorragia, anemia, entre outras complicações. Caso o RN sugue a mama antes de a placenta sair, a ocitocina liberada pela amamentação pode acelerar a dequitação placentária, além de ajudar na "descida do leite" ${ }^{11}$.

Aponta-se que nem todo o recém-nascido está pronto para sugar imediatamente após o parto, ou nem toda mãe está pronta para amamentar imediatamente após o parto, no entanto, é importante que esse RN seja colocado em contato direto pele a pele no abdômen/tórax da mãe, se ambos estiverem em boas condições de saúde e se esse for o desejo da mulher. Estudos comprovam que o contato pele a pele logo após o parto faz o recém-nascido se adapte mais
Ramiro NCMP, Pereira MS, Souza RS, Chaparin BRM, Navarro BVA, Aver LA rapidamente à vida extrauterina, além de promover o vínculo mãe-bebê e auxiliar no estabelecimento da amamentação. Além disso, esse contato íntimo, pele a pele, entre mãe e filho faz com que a mãe passe para o bebê os micróbios de sua pele, que vão protegê-lo contra infecções ${ }^{12}$.

Existem diversos fatores protetores da amamentação na primeira hora de vida, tais como: condições materna e neonatal saudáveis, a realização adequada do pré-natal, presença de alojamento conjunto, cuidados de enfermagem englobando o binômio mãe-bebê, a presença de orientações prévias acerca dos benefícios do aleitamento materno, e o desejo e planejamento da gravidez. Entretanto, cita-se a realização inadequada do prénatal, realização de cesariana, número de profissionais insuficientes, ausência de alojamento conjunto, baixo peso ao nascer e a desinformação da gestante como fatores que dificultam a prática do ato de amamentar na primeira após o parto $7,10,13,18$.

\section{Conclusão}

Com base nos estudos apresentados podemos concluir que o aleitamento materno na primeira hora de vida é de extrema importância, gerando diversos benefícios como uma redução significativa do índice de mortalidade neonatal, proteção imunológica através da produção de anticorpos proveniente do colostro, também conhecida como "vacina natural", estabelecer o vínculo maternoinfantil, a troca de sentimentos entre a mãe e o recémnascido, promove a regulação da glicemia e auxiliando sistema cardiorrespiratório, e assim auxilia na adaptação extrauterina do recém-nascido. Um benefício importante para a mãe se dá pela produção de ocitocina, reduzindo o risco de se desenvolver hemorragia e outras complicações. Contudo para que isso possa ser realizado é importante garantir que as condições sejam favoráveis, visto que, em alguns casos, a mãe e o bebê não estão prontos ou não apresentam condições para realizar a amamentação.

\section{Referências}

1. Hergessel NM, Lohmann PM. Aleitamento materno na primeira hora após o parto. Centro Universitário Univates, Lajeado/RS, 2018. Disponível em: https://www.univates.br/bdu/bitstream/10737/1785/1/2017NadirMariaHergessel.pdf

2. Paredes HDMT, et al. Amamentação na primeira hora de vida em uma maternidade de referência de Macaé. Saúde Redes [Internet]. 2019;5(1):35-47. Disponível em: http://revista.redeunida.org.br/ojs/index.php/rede-unida/article/view/1820

3. Rocha AF, Gomes KRO, Rodrigues MTP. Impacto da intenção de engravidar sobre a amamentação na primeira hora pós-parto. Ciência \& Saúde Coletiva. 2020;25(10):4077-4086.

4. Oliveira LF. Conhecimento das puérperas sobre os benefícios da amamentação em ambiente hospitalar. Cadernos da Escola de Saúde [Internet]. 2018;18(1):1-22. Disponível em: https://portaldeperiodicos.unibrasil.com.br/index.php/cadernossaude/article/view/3784

5. Sousa LMM, et al. A metodologia de revisão integrativa da literatura em enfermagem. Revista investigação em enfermagem [Internet]. 2017;21:17-26. Disponível em: https://www.scielo.br/j/eins/a/ZQTBkVJZqcWrTT34cXLjtBx/?format=pdf\&lang=pt

6. ROCHA, Letícia Braga et al. Aleitamento materno na primeira hora de vida: uma revisão da literatura. Revista de Medicina e Saúde de Brasília [Internet]. 2017;6(3). Disponível em: https://portalrevistas.ucb.br/index.php/rmsbr/article/view/8318

7. Jesus AS, et al. Amamentação na primeira hora de vida entre mulheres do Nordeste brasileiro: prevalência e fatores associados. Revista Eletrônica de Enfermagem [Internet]. 2020;22. Disponível em: https://revistas.ufg.br/fen/article/view/58772

8. Santos RPB, et al. Importância do colostro para a saúde do recém-nascido: percepção das puérperas. Rev. enferm. UFPE on line [Internet]. 2017;11(Supl.9):3516-3522. Disponível em: https://pesquisa.bvsalud.org/portal/resource/pt/biblio-1032538 
Os benefícios do aleitamento materno na primeira hora de vida Ramiro NCMP, Pereira MS, Souza RS, Chaparin BRM, Navarro BVA, Aver LA

9. Leite MFFS, et al. Promoção do aleitamento materno na primeira hora de vida do recém-nascido por profissionais da enfermagem. Arquivos de Ciências da Saúde da UNIPAR [Internet]. 2016;20(2). Disponível em:

https://revistas.unipar.br/index.php/saude/article/view/5386

10. Silva JLP, et al. Fatores associados ao aleitamento materno na primeira hora de vida em um hospital amigo da criança. Texto \& ContextoEnfermagem. 2018;17(4). https://doi.org/10.1590/0104-07072018004190017

11. Sena RP, et al. Ação educativa para as gestantes na promoção da "Golden hour": relato de experiência. Revista Eletrônica Acervo Saúde [Internet]. 2020;40:e2291. Disponível em: https://acervomais.com.br/index.php/saude/article/view/2291

12. Ramalho AA, et al. Fatores associados à amamentação na primeira hora de vida em Rio Branco, Acre. DEMETRA: Alimentação, Nutrição \& Saúde [Internet]. 2019;14:43809. Disponível em: https://www.e-publicacoes.uerj.br/index.php/demetra/article/view/43809

13. Sampaio ARR, Bousquat $A$, Barros $C$. Contato pele a pele ao nascer: um desafio para a promoção do aleitamento materno em maternidade pública no Nordeste brasileiro com o título de Hospital Amigo da Criança. Epidemiologia e Serviços de Saúde [Internet]. 2016;25(2):281-290. Disponível em: https://doi.org/10.5123/S1679-49742016000200007

14. Antunes $\mathrm{MB}$, et al. Amamentação na primeira hora de vida: conhecimento e prática da equipe multiprofissional. Avanços em Enfermagem [Internet]. 2017;35(1):19-29. Disponível em: https://pesquisa.bvsalud.org/controlecancer/resource/pt/biblio-888398?src=similardocs

15. Rodrigues CSF, et al. Aleitamento materno exclusivo na primeira hora de vida: uma revisão integrativa. Research, Society and Development [Internet]. 2020;9(7):e799974799. Disponível em: https://rsdjournal.org/index.php/rsd/article/view/4799

16. Jung SM, Rodrigues FA, Herber S. Contato pele a pele e aleitamento materno: experiências de puérperas. Revista de Enfermagem do Centro-Oeste Mineiro [Internet]. 2020;10. Disponível em: http://www.seer.ufsj.edu.br/index.php/recom/article/view/3657

17. Terra NO, et al. Fatores intervenientes na adesão à amamentação na primeira hora de vida: revisão integrativa. Revista Eletrônica de Enfermagem [Internet]. 2020;22. Disponível em: https://revistas.ufg.br/fen/article/view/62254

18. Garcia FM, Rosa T. Assistência de enfermagem frente aos transtornos na lactação: uma revisão integrativa. Glob Acad Nurs. 2020;1(2):e29. https://doi.org/10.5935/2675-5602.20200029 